\title{
Particle physics after the Higgs discovery: philosophical perspectives
}

\author{
Simon Friederich \\ email@simonfriederich.eu
}

University of Groningen,

University College Groningen, Hoendiepskade 23/24, NL-9718 BG Groningen,

Faculty of Philosophy, Oude Boteringestraat 52, NL-9712 GL Groningen, The Netherlands

\author{
Dennis Lehmkuhl \\ lehmkuhl@caltech.edu \\ California Institute of Technology \\ Einstein Papers Project and Division of the Humanities and Social Sciences \\ Caltech M/C 20-7, 1200 East California Blvd. \\ Pasadena, CA 91125, USA
}

\section{Introduction}

The recent discovery at the LHC of a particle with properties matching those expected of the Higgs boson is a decisive event in the history of particle physics. The present special section combines three contributions that approach conceptual and methodological challenges related to this event and the current situation in particle physics from different angles. One contribution studies the experimental practices of contemporary particle physics by investigating the role of computer simulations in these practices; in particular, it focuses on the status of simulations as compared to experiments that in some circumstances have analogous functions. One contribution investigates the status of the controversial naturalness problem that many physicists see as the most severe shortcoming of the Standard Model of elementary particle physics. Finally, a third contribution critically assesses the impact of suggested no-go theorems concerning the interpretability of rigorous algebraic quantum field theory in terms of particles at the phenomenological level. In what follows we present a short overview of these contributions, highlighting some of their central ideas and arguments and putting them into context.

\section{Overview of the contributions}

\subsection{Simulations and experiments}

The contribution by Michela Massimi and Wahid Bhimji highlights crucial features of the 
experimental context in which discoveries in particle physics such as that of the Higgs particle are made. They do so by elucidating the status of computer simulations in these discoveries. Simulations have come to play a crucial and ineliminable role in all areas of physics and in recent years there has been a lively philosophical debate concerning the significance and legitimacy of this role.

Philosophers have focused, in particular, on the comparison of simulations and ordinary experiments as alternative, potentially rival, means of acquiring knowledge about some physical target system. Various authors (e.g. Guala 2005, Morgan 2005, Giere 2009) have called for caution concerning the representational and inferential trustworthiness of simulations as compared to experiments by endorsing claims of epistemic priority of experiments over simulations. In particular, these authors claim that simulations lack a crucial feature that experiments have, a feature that enables one to draw reliable inferences concerning the target system under study. Namely, unlike ordinary experiments, simulations do not involve a causal interaction with the target system. According to the critics mentioned, this feature of simulations severely limits the trustworthiness of claims concerning the representational adequacy of the models used in the simulations and significantly constrains the inferences that can be reliably drawn from the simulations.

Massimi and Bhimji attack the thesis of the epistemic priority of experiments over simulations by criticizing a core assumption that is shared by all its defenders: the assumption that simulations do not involve causal interaction with their target physical systems whereas ordinary experiments do. To assess this assumption, Massimi and Bhimji carefully distinguish between various ways in which experiments involve causal interaction with target systems, i.e. different ways in which simulations might lack some crucial feature of experiments. In a case study concerning the use of simulations in various stages of the ATLAS experiment at the LHC they find that simulations do in fact involve causal interactions with target systems in all the ways specified. In particular, they point out that simulations play key roles in calibration, in the tracking of causal interactions and in causal inferences concerning the existence of hypothesized entities. These observations, they suggest, speak against the view that simulations lack causal interaction with target systems. Furthermore, they observe that where simulations compete directly with experiments as a means of extracting the same information, e.g. in background detection, simulations often provide the much-needed higher accuracy. Massimi and Bhimji take this to support their case against the epistemic priority of experiments over simulations.

\subsection{Naturalness}

The fact that the Standard Model of elementary particle physics violates a criterion commonly called naturalness is widely regarded as one of its most serious shortcomings by particle physicists; indeed, perhaps as its most serious shortcoming. In the Standard Model, the violation of naturalness arises from the so-called hierarchy problem: the fact that the electroweak scale is much smaller than the scale where the Standard Model breaks down (possibly as large as the Planck scale) and hitherto unknown physics is expected to set in. This problem is connected directly to the Higgs mechanism (it is equivalent to the problem of why the Higgs mass is so much smaller than the Planck mass), and worries about it underlie many 
worries about the Higgs mechanism being merely an ad hoc solution to the problems of mass generation that it was designed to solve (Friederich et al. 2014). Attempts to overcome this hierarchy problem by developing models and theories that conform to naturalness have long dominated theoretical high energy physics. In particular, this criterion is one of the driving forces behind searches for so-called supersymmetric partner particles of known elementary particles, which—should they be revealed to exist—might dispel the naturalness problem or at least make it much less severe.

Given its enormous influence, significance and conceptual intricacy, the criterion of naturalness has attracted surprisingly little attention from philosophy of science. Porter Williams' paper Naturalness, the autonomy of scales, and the $125 \mathrm{GeV}$ Higgs provides both an analysis of competing accounts of what exactly the naturalness problem amounts to, and an outline of the problem's ontological ramifications. The notion of naturalness that Williams singles out as the one which captures best how physicists actually use the naturalness criterion is centred around the idea that in natural theories the low-energy physical phenomena should not depend sensitively on high-energy phenomena. Williams compares this account of naturalness to rival accounts, which either tie naturalness to quadratic divergences in the renormalization flow, construe it as a symmetry principle, interpret is as an anti-fine-tuning condition or denigrate its status to a merely aesthetic criterion. Williams argues that none of these other accounts of naturalness can do justice to its actual application, nor to what are considered worrisome violations of naturalness in physical practice.

Williams outlines the ontological ramifications of the breakdown of naturalness associated with the hierarchy problem. He argues that this breakdown gives us strong hints against the conception of reality as a layered hierarchy of "quasi-autonomous domains", as advocated in Cao and Schweber (1993, p. 72). According to Williams, since the Higgs mass exhibits an extremely strong dependence on the details of whatever occurs at extremely high energies, quasi-autonomy as claimed by Cao and Schweber does not seem to obtain.

In view of its violation in the Standard Model of elementary particle physics, might it not be a good idea to abandon naturalness altogether as a principle of theory choice? Williams argues that long-standing experiences, in particular the success of the effective field theories viewpoint, lead one to expect that successful theories in particle physics will indeed conform to naturalness, and he highlights the support that this expectation receives from a formal result known as the decoupling theorem (Applelquist and Carazzone 1975).

Williams' considerations sit well with considerations recently put forward by James Wells in this journal (Wells 2015). Wells argues for a kind of naturalness that can be seen as an instance of optimistic meta-induction: Starting from a naturalness problem that arises from the smallness of the electron mass as compared to the mass scale set by Fermi's theory of the weak interaction, Wells suggests a series of compelling inferential steps of modifying the theory that culminate in the introduction of a scalar boson with exactly the features of the Higgs boson. Thus, according to Wells, many historical steps towards the Standard Model that turned out to be empirically valid might have been taken based on naturalness alone as a guide in theory building. The considerations offered by Williams and Wells may be taken to suggest that one should not abandon naturalness as a guide in theory choice, even though in the present situation of particle physics there is hardly any empirical support for straightforwardly natural alternatives to the Standard Model. 


\subsection{Particle phenomenology}

Modern theories of particle physics are formulated in the language of quantum field theory. It is therefore prima facie disturbing that there are various no-go theorems that seem to rule out interpreting quantum field theories as incorporating the constraint of relativistic causality in terms of localizable particles. How can these results be reconciled with the spectacular empirical successes of modern particle physics as the discovery of the Higgs particle?

A step towards an answer to this question was made in an influential paper by Halvorson and Clifton (2002). The paper not only reviewed and generalized the no-go theorems on particle interpretations and proved them explicitly within the framework of the mathematically rigorous algebraic framework for quantum field theories, but at the same time assessed their scope and relevance. Halvorson and Clifton pointed out that, even though algebraic quantum field theories do not permit an interpretation in terms of a particle ontology, there may still be room to accommodate for apparent particle phenomenology in these theories, to a degree that allows compatibility with the manifest empirical facts. Halvorson and Clifton base their conciliatory strategy concerning the no-go theorems on the evocation of almost (rather than fully) local observables.

Somewhat disturbingly, Arageorgis and Stergiou (2013) recently posed a new challenge to the conciliatory strategy, based on a novel result in the algebraic approach. They interpret the result as demonstrating an essential incompatibility of relativistic causality and almost local observables, and argue that particle detectors that implement these observables would lead to act-outcome correlations across the entire space-time. Thus, they would be in conflict with an interpretation in terms of sufficiently localizable particles.

Giovanni Valente's contribution to this special section assesses the significance of the result by Arageorgis and Stergiou. He argues that, on closer inspection, there is no conflict between Arageorgis' and Stergiou's result and the idea of approximately localized observables. The apparent threat to particle phenomenology can be diffused, Valente argues, if one recognizes that almost local observables are not genuine observables inasmuch as acts of measurement always take place within bounded, “local”, space-time regions. The observables that pertain to these regions all obey the relativistic causality principle, ensuring that there can be no act-outcome correlations across space-time for the results of their measurement. The appeal to almost local observables nevertheless suffices to preserve a particle phenomenology in that measurement of local observables can mimick measurement of almost local observables to arbitrary degrees of accuracy.

Valente concludes his paper by noting that even though the door to reading algebraic quantum field theory as licensing particle phenomenology remains open, the door to the foundational challenge of specifying a suitable ontology for algebraic quantum field theory remains open as well.

\section{References}

Appelquist, T. and Carazzone, J. (1975): Infrared singularities and massive fields, Physical Review D, 11:2856-2861.

Arageorgis, A. and Stergiou, C. (2013), On particle phenomenology without particle ontology: 
How much local is almost local?, Foundations of Physics, 43:969-977.

Cao, T.Y. and Schweber, S. (1993), The conceptual foundations and the philosophical aspects of renormalization theory, Synthese, 97:33-108.

Friederich, S., Harlander, R., and Karaca, K (2014), Philosophical perspectives on ad hoc hypothe- ses and the Higgs mechanism, Synthese, 191:3897-3917.

Giere, R. (2009), Is computer simulation changing the face of experimentation?, Philosophical Studies 143:59-62.

Guala, F. (2005), Models, simulations, and experiments, in: L. Magnani and N. Nersessian (eds.), Model-based Reasoning: Science, Technology, Values, Kluwer, pp. 59-74.

Halvorson, H. and Clifton, R. (2002), No place for particles in relativistic quantum theories?, Philosophy of Science, 69:1-28.

Morgan, M. (2005), Experiments versus models: new phenomena, inference, and surprise, Journal of Economic Methodology, 12:317-29.

Wells, J. D. (2015), The utility of Naturalness, and how its application to Quantum Electrodynamics envisages the Standard Model and Higgs boson, Studies in History and Philosophy of Modern Physics, 49:102-108. 